\title{
Tachycardia and Bradycardia Coexisting in the Same Pulmonary Vein
}

\author{
HAKAN ORAL, M.D., BRADLEY P. KNIGHT, M.D., \\ HIROSHI TADA, M.D., and FRED MORADY, M.D.
}

From the Division of Cardiology, Department of Internal Medicine, University of Michigan, Ann Arbor, Michigan

\begin{abstract}
Dissociated Pulmonary Vein Rhythms. During segmental isolation of the pulmonary veins (PVs) in a patient with paroxysmal atrial fibrillation, there was a PV bradycardia that was dissociated from the left atrium in a segment of the right superior PV, whereas the remaining segments showed passive activation of a PV fascicle during sinus rhythm. Rapid atrial pacing induced a PV tachycardia in the nonisolated fascicles, and the dissociated PV bradycardia persisted in a segment of the same PV. These observations indicate that PV fascicles are insulated from each other and that a dissociated PV rhythm does not necessarily indicate complete isolation of a PV. ( Cardiovasc Electrophysiol, Vol. 13, pp. 186-188, February 2002)
\end{abstract}

atrial fibrillation, pulmonary vein isolation, radiofrequency catheter ablation, pulmonary vein rhythms

\section{Introduction}

In patients with atrial fibrillation (AF), segmental isolation of pulmonary veins (PVs) guided by a circular mapping catheter has been performed to electrically disconnect the PVs from the left atrium. ${ }^{1}$ During isolation of PVs, a rhythm within a PV that is dissociated from the left atrium has been considered indicative of complete electrical isolation of the PV. ${ }^{2}$ This case report demonstrates that a dissociated PV rhythm does not necessarily indicate complete isolation of a PV.

\section{Case Report}

In a 45-year-old man with paroxysmal AF, segmental isolation of the PVs guided by ostial potentials was performed. AF was first diagnosed 8 years prior to presentation. The patient experienced approximately 30 episodes of AF per month despite antiarrhythmic drug therapy. He had no structural heart disease.

\section{Segmental PV Isolation}

After informed consent was obtained, a PV isolation procedure was performed. With a right femoral vein approach, a quadripolar electrode catheter (EP Technologies, Inc., San Jose, CA, USA) was positioned in the distal coronary sinus. Transseptal catheterization was performed, and a deflectable, decapolar catheter with a distal ring configuration (Lasso catheter; Biosense Webster, Diamond Bar, CA, USA) and a deflectable, quadripolar 7-French catheter with $2-5-2 \mathrm{~mm}$ interelectrode spacing and a 4-mm distal electrode with an embedded thermistor (EP Technologies) were inserted into the left atrium. An activated clotting time of 250 to 350 seconds was maintained throughout the case with heparin. Angiograms of the left superior (LS), right superior (RS), and left inferior (LI) PVs were performed to identify the PV ostia. Bipolar electrograms were filtered at bandpass settings of 30 to $500 \mathrm{~Hz}$ and recorded digitally (EPMed Systems, Inc., Mount Arling-

Address for correspondence: Hakan Oral, M.D., Division of Cardiology, Department of Internal Medicine, University of Michigan, 1500 E. Medical Center Drive, B1F245, Ann Arbor, MI 48109-0022. Fax: 734-936-7026; E-mail: oralh@umich.edu

Manuscript received 30 September 2001; Accepted for publication 27 November 2001. ton, NJ, USA). Atrial pacing was performed from the coronary sinus with a stimulator (model EP-3 Clinical Stimulator; EPMed Systems).

The Lasso catheter was positioned within $5 \mathrm{~mm}$ of the PV ostium. PV isolation was performed during sinus rhythm or coronary sinus pacing and was guided by ostial PV potentials. Radiofrequency energy was delivered within $5 \mathrm{~mm}$ of the PV ostium as individual 30- to 60-second applications, with a target temperature of $52^{\circ} \mathrm{C}$ and a maximum power output of 30 to 35 W (model EPT-1000-TC; EP Technologies). Elimination of all ostial PV potentials and complete entrance block into the PV were considered indicative of complete electrical isolation of the PV. The LS, LI, and RS PVs were targeted for PV isolation.

\section{PV Tachycardia and Bradycardia Within the Same PV}

The LS and LI PVs were the first veins isolated. During radiofrequency energy application at the RS PV ostium, a PV bradycardia with a cycle length of 2,600 msec that was dissociated from the other segments of the $P V$ and from the left atrium was recorded by 4 of the 10 electrode pairs of the Lasso catheter (Fig. 1). Discrete, high-frequency PV potentials resulting from passive activation during sinus rhythm were recorded by the remaining 6 of 10 electrode pairs. AF was induced by rapid atrial pacing, and a PV tachycardia was recorded by 6 of the 10 electrode pairs, whereas the dissociated PV bradycardia persisted at the other electrode pairs (Fig. 2).

By targeting the remaining PV fascicle, the RS PV was completely isolated. The dissociated PV rhythm resolved within 10 minutes after isolation.

\section{Discussion}

This case report demonstrates that a bradycardia and tachycardia can coexist in the same PV. This implies that the fascicles that make up the muscle sleeves that surround the PVs are electrically insulated from each other. Furthermore, the observations in this case report demonstrate that a $\mathrm{PV}$ rhythm that is dissociated from the left atrium does not necessarily indicate complete electrical isolation of a PV. Therefore, complete elimination of all ostial PV potentials with entrance block into the PV should be the endpoint of segmental PV isolation.

After segmental isolation, rhythms that are dissociated from the left atrium are observed within $\sim 10 \%$ of PVs. ${ }^{2}$ 
Figure 1. Example of pulmonary vein $(P V)$ bradycardia (cycle length 2,600 msec) that is dissociated from the remaining fascicles of the $P V$ and the left atrium. Shown are leads I and V5 and intracardiac electrograms recorded by the Lasso catheter (L1-2 to L10-1) and the coronary sinus catheter (CS). The Lasso catheter was positioned in the right superior PV (arrows), within 5 $\mathrm{mm}$ of the ostium. The bradycardia is recorded at poles 6-7 to 9-10. Passive $P V$ potentials resulting from sinus rhythm are present at the remaining electrode bipoles (asterisks).
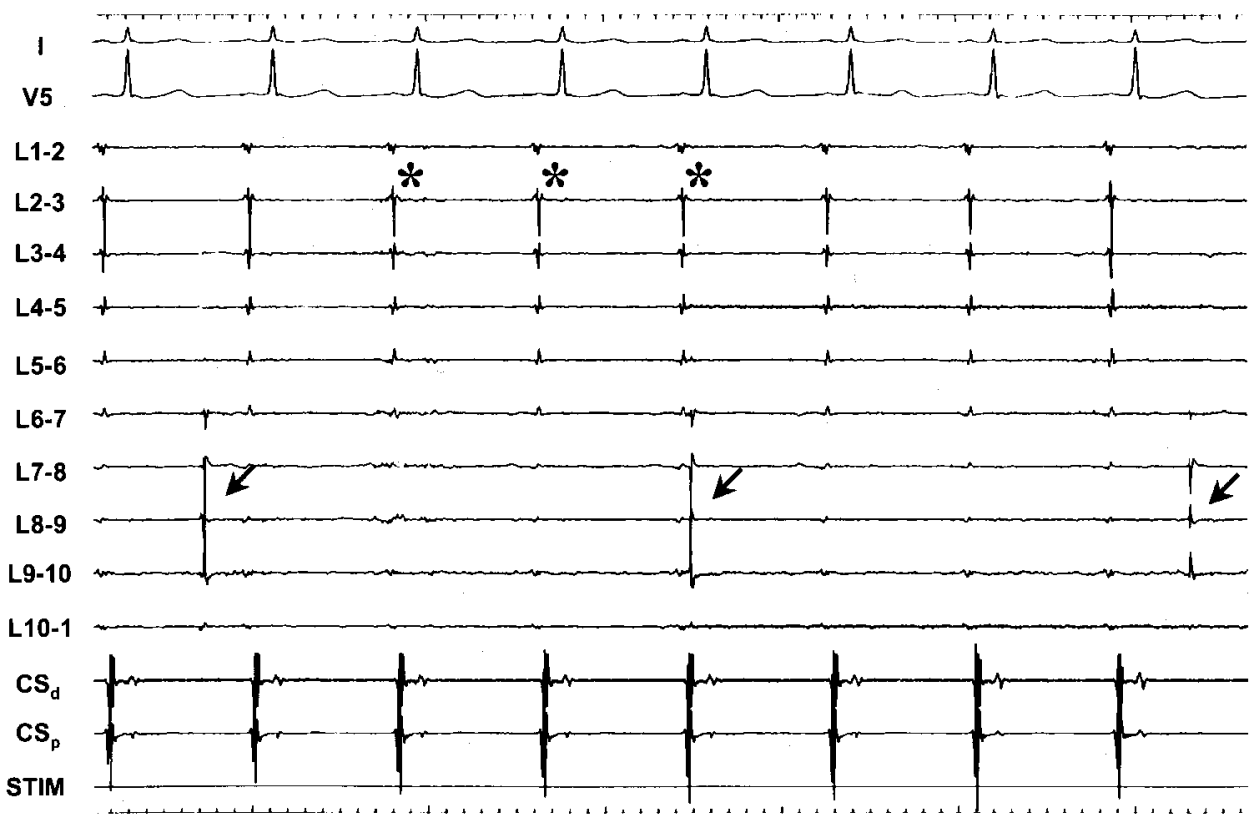

$1 \mathrm{sec}$
More than $95 \%$ of these dissociated rhythms are PV bradycardias; the remaining are tachycardias.

Myocardial sleeves that surround the PVs are not continuous. They show a complex, multilayer, three-dimensional organization. ${ }^{3}$ Fibrous connective tissue may provide a conduction barrier among the myocardial sleeves, rendering PV fascicles electrically insulated.

In a prior study, PV isolation was performed guided by localization of PV fascicles with a noncontact map- ping system. ${ }^{4}$ However, this report underscores the importance of high-density mapping of the entire circumference of PVs, which may not be feasible with a noncontact mapping system due to limitations of the spatial resolution.

In summary, this report provides evidence that PV fascicles are insulated; therefore, the whole circumference of the PV ostia should be mapped carefully to confirm complete isolation.
Figure 2. Example of pulmonary vein $(P V)$ bradycardia and tachycardia coexisting in the same PV. Atrial fibrillation was induced by rapid atrial pacing, and a tachycardia (cycle length $154 \mathrm{msec}$ ) was recorded at poles 1-2 through 6-7, whereas the bradycardia persisted at poles 6-7 to 9-10. Catheter positions and abbreviations as in Figure 1.

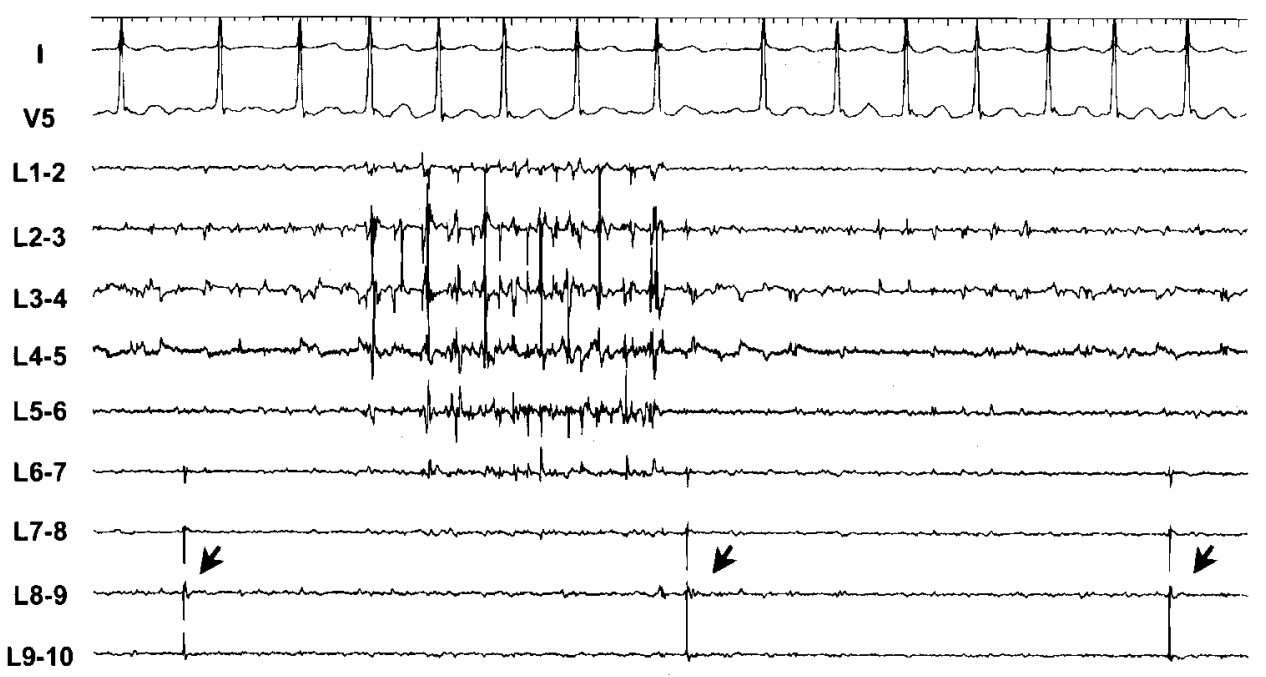

L10-1

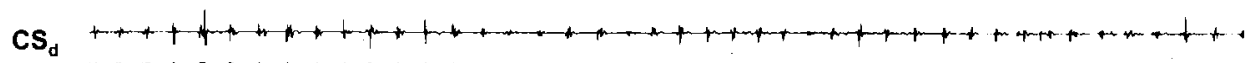

cs. Hetw STIM $1 \mathrm{sec}$ 


\section{References}

1. Haissaguerre M, Shah DC, Jais P, Hocini M, Yamane T, Deisenhofer I, Chauvin M, Garrigue S, Clementy J: Electrophysiological breakthroughs from the left atrium to the pulmonary veins. Circulation 2000;102:2463-2465

2. Jais P, Shah D, Salerno J, Hocini M, Yamane T, Deisenhofer I, Clementy J, Haissaguerre M: Dissociation of venous activity following radiofrequency ablation of pulmonary vein ostia for atrial fibrillation. (Abstract) Circulation 2000;102:II-484.

3. Saito T, Waki K, Becker AE: Left atrial myocardial extension onto pulmonary veins in humans: Anatomic observations relevant for atrial arrhythmias. J Cardiovasc Electrophysiol 2000;11:888-894.

4. Hindricks G, Kottkamp H: Simultaneous noncontact mapping of left atrium in patients with paroxysmal atrial fibrillation. Circulation 2001; 104:297-303 